\title{
Introducing a non-invasive monitoring approach for bridge infrastructure with ground-based interferometric radar
}

\author{
Chris Michel (D) *a and Sina Keller (D) a \\ ${ }^{a}$ Karlsruhe Institute of Technology (KIT) - Institute of Photogrammetry and Remote Sensing \\ Kaiserstr. 12, 76131 Karlsruhe (Germany) \\ *Correspondence: chris.michel@kit.edu
}

\begin{abstract}
In this paper, we introduce a non-invasive approach for monitoring bridge infrastructure with ground-based interferometric radar. This approach is called the mirror mode, since it utilises the flat surface of the bridge underside as a mirror to reflect the signal to a corner reflector on the ground placed opposite of the radar sensor. For proving the feasibility of this approach, a measurement campaign has been carried out at an exemplary bridge in Karlsruhe (Germany) including a radar sensor in mirror mode, a second radar sensor in the default mode and a laser profile scanner. We investigate the potential of this approach to monitor the bridge displacement in vertical direction and compare the results with the two other sensors. The derived results reveal the potential for monitoring bridge infrastructure. Finally, we propose further research aspects of this approach to analyse its capabilities and limitation in the context of non-invasive infrastructure monitoring.
\end{abstract}

Keywords: Displacement monitoring, ground-based interferometric radar, non contact measurement, structural health monitoring (SHM)

\section{Introduction}

Bridges are an important part of the critical transport infrastructure, hence their condition needs to be periodically assessed. One part of this assessment is monitoring of bridge behaviour in operation and over time. Changes in this behaviour such as varying displacement or strain response caused by static or dynamic loads can indicate structural deteriorations or damages. Traditionally, the dynamics of bridges are monitored with strain gauges and accelerometers. At this point, remote sensing techniques such as ground-based interferometric radar (GBR) show great potential to be used instead to avoid the complex installation of directly contacting sensors. GBR is able to measure the displacement in line of sight (LOS) with precision in the sub-millimetre range at a sample frequency of up to $200 \mathrm{~Hz}$ [1]. It has been applied to different infrastructures (see e.g. [2]). In the context of measuring the displacement of bridge infrastructure, transversal beams at the bridge underside are widely used as reflective targets for the radar signal. A valid alternative to ensure sufficient signal reflection is the application of corner reflectors. These reflectors need to be mounted at the bridge which can be hard or sometimes impossible to accomplish, depending on the construction of the bridge. Their installation can also interfere with normal operation of the bridge and increases setup time. In addition, the use of reflectors does not support the principle of contactless measurement, which is a key advantage of monitoring with GBR.
In this contribution, we propose a measurement approach which is detached from using scattering points or reflectors at the bridge. This non-invasive approach utilises the flat surface of the lower side of the bridge deck as a mirror [3]. The radar signal is reflected towards the ground. Then, the GBR receives the reflected signal on the same path as the emitted signal. Thus, the displacement of the mirror point can be measured.

An essential prerequisite to rely on this measurement setup is given by the bridge construction: it has to exhibit a flat underside. Since a large number of small and medium sized highway bridges in Germany are characterized by a flat underside, most of these bridges are not monitored contactlessly due to the necessary installation of reflectors. Thereby, they are predestined for the proposed fast and practicable measurement approach.

Overall, the main contributions of this paper are:

- a detailed description of the working principle of the non-invasive measurement approach based on GBR;

- an application at a bridge including a second GBR and a laser profile scanner as two additional sensors for comparison;

- a comprehensive evaluation of the proposed measurement approach and an analysis of its potential in regards to the monitoring of bridge infrastructure;

- a discussion of arising challenges compared to the default operation of GBR during bridge monitoring. 


\section{Setup and methodology}

\subsection{Measurement with GBR: state of the art}

A GBR measures the differential displacement by detecting phase differences in the backscattered signal. A modulation of frequency allows to distinguish scatterers in varying range cells [1]. In default operation when monitoring bridge infrastructure, the GBR is positioned below the bridge facing the underside at an angle. Therefore, displacements of several scattering points can be measured at once. As the GBR only detects displacement differences in LOS, the measurements have to be projected to a common axis for comparing the results. In general, the vertical axis is used, since it is assumed that a bridge has the largest displacement amplitude in this direction. The precision of the measurement is correlated with the signal to noise ratio (SNR) of the backscattered signal. It is typically lower than $0.1 \mathrm{~mm}$.

\subsection{The mirror mode as an alternative mea- surement approach}

A large number of bridges exhibit flat surfaces at their underside. These surfaces do not reflect the signal back to the sensor and corner reflectors would have to be installed. Instead we use the underside as a mirror to generate a secondary reflection at the ground opposite of the GBR (see [3]). From the ground the signal is reflected back on the same path as visualized in Figure 1. To ensure high enough $\mathrm{SNR}$, a corner reflector is placed on the ground facing the bridge underside. Similar to the default operation, the LOS measurement has to be projected to the vertical axis. The projected displacement $d$ results from the LOS measurement $d_{L}$, with the coordinates $\left(x_{1}, y_{1}\right)$ of the respective mirror point:

$$
d=\frac{d_{L} \sqrt{x_{1}^{2}+y_{1}^{2}}}{2 y_{1}}
$$

In contrast to the default operation, the factor $1 / 2$ is added, since the GBR measures twice the displacement in LOS as is shown in Figure 1. The coordinates of the mirror point are unknown but can be calculated with some assumptions on the measurement setup. As the incidence angle is equal to the angle of reflection, the following relation is defined as

$$
\frac{y_{1}}{x_{1}}=\frac{y_{2}}{x_{2}-x_{1}}
$$

Assuming the $\mathrm{X}$-axis of the local coordinate system is parallel to the bridge underside, then the $y$-axis values are constant and can be measured. Furthermore, the distance $x_{2}$ between GBR and reflector is also known. Therefore, the position of the mirror point $x_{1}$ results from

$$
x_{1}=\frac{x_{2} y_{1}}{y_{1}+y_{2}}
$$

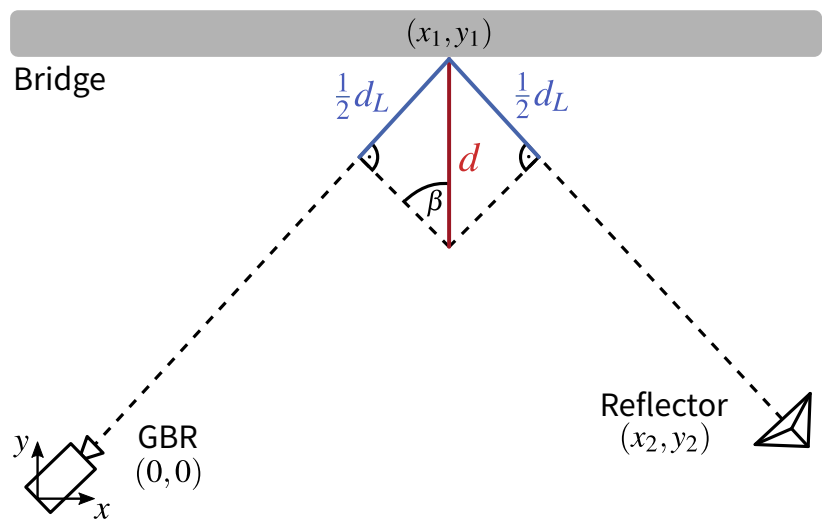

Figure 1 Setup of the projection in mirror mode.

In default operation it is assumed that the horizontal displacement of the bridge is negligible in comparison to the vertical displacement. According to [4], this assumption is not always applicable. For measurement points located further away from the centre of the span, a significant influence of the horizontal component was observed. As the GBR always measures the sum of both components in LOS, they cannot be differentiated. By using the bridge underside as a mirror only the vertical component is measured; other components do not influence the measurement.

Compared to the default operation, the measurement in mirror mode is expected to have a lower SNR, because the signal is reflected more than once. According to [6], the measurement precision is correlated with the SNR

$$
\sigma_{d_{L}}=\frac{\lambda}{4 \pi \cdot \sqrt{2 \cdot \mathrm{SNR}}}
$$

where $\lambda$ is the wavelength of the GBR. From the context of variance propagation, the mirror mode has an advantage to the default operation. Usually the variance increases, when the displacements are projected to the vertical axis. In mirror mode the projection decreases the variance, since twice the displacement is measured.

\section{Application and data processing}

To evaluate the approach described above, a measurement campaign was carried out at a bridge in Karlsruhe, Germany. This four-span prestressed concrete bridge has three lanes for one way of car and truck traffic.

\subsection{Measurement setup}

The sensor and a reflector were placed under one of the concrete beams as shown in Figure 2. The point, at which the displacement is measured, is called the mirror point. This point is located approximately in the middle of the span. To evaluate and analyse the potential of the proposed measurement approach, we applied a second GBR and a laser profile scanner during the measurement campaign. Figure 3 shows the entire setup with the sensors and reflectors. The second GBR was placed directly under 
the mirror point. As this sensor is aligned with the vertical axis, no projection is needed. The displacement measured in LOS is equivalent to the vertical displacement.

The laser profile scanner uses a rotating mirror to produce two dimensional point clouds with a repetition rate of up to $200 \mathrm{~Hz}$. By positioning the laser profile in line with the concrete beam, the scanner is able to measure the vertical displacement of the whole beam. For the evaluation and direct comparison between the GBR in mirror mode and the profile scanner, a small subset of the profile at the mirror point is analysed. Table $\mathbf{1}$ summarises the respective sensor specifications.

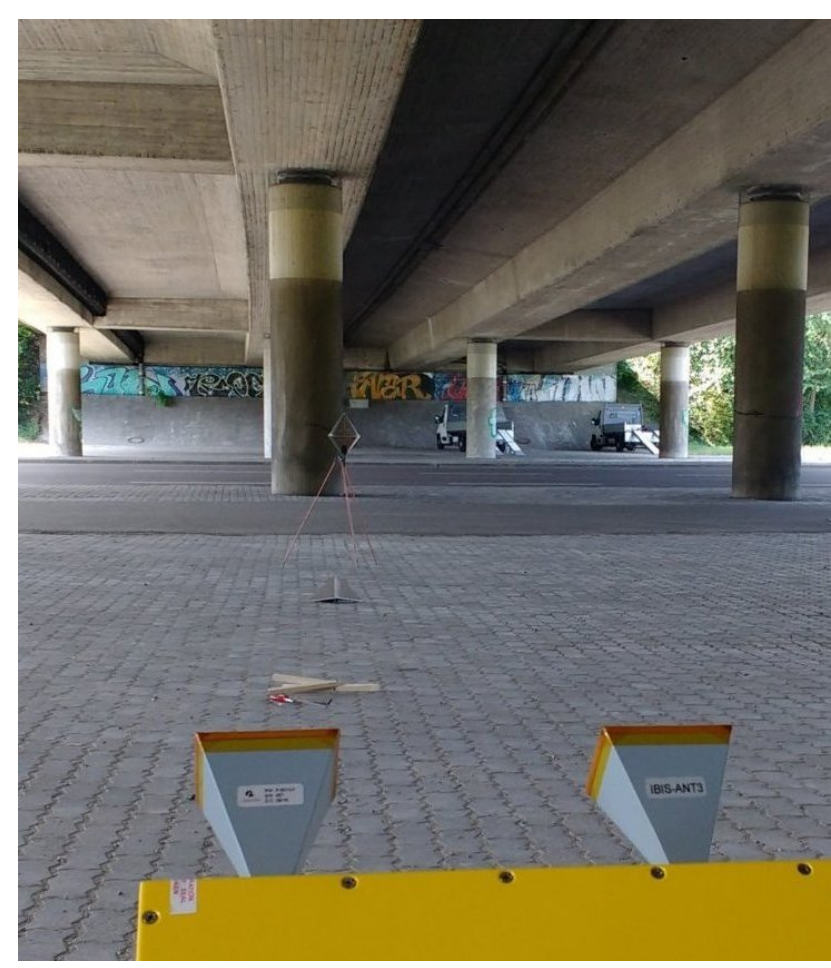

Figure 2 Setup of the GBR in mirror mode with a reflector under a concrete beam.

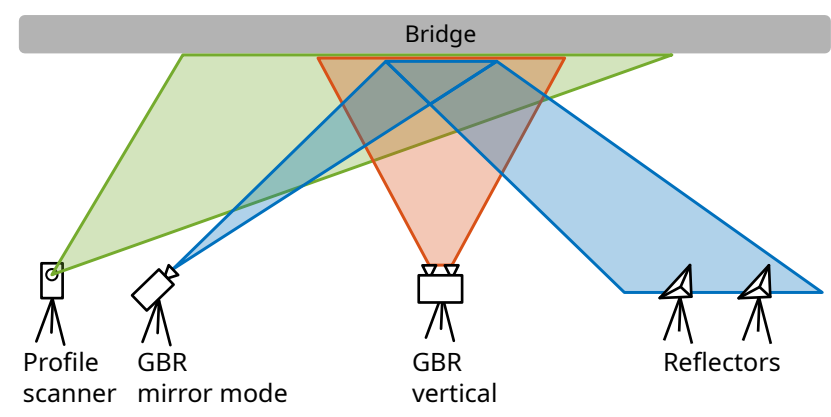

Figure 3 Setup of the three applied sensors.

\subsection{Data processing}

A direct comparison of the measured time series requires sensor specific processing steps. For the two GBR these steps are largely the same (see Figure 4). The sensors measure the in-phase and quadrature components of the received signal in the time-frequency domain. These com- ponents are transformed to the time-range domain with an Inverse Discrete Fourier Transform (IDFT). Amplitude and phase are calculated with the absolute and the argument respectively. The displacement in LOS results from unwrapping the phase and is projected to a common axis depending on the measurement geometry.

An additional processing step of clutter removal has to be implemented for the GBR in mirror mode. As the GBR only has range resolution, it measures the sum of all displacements in the respective range cells. Scattering points moving in different directions in the same range cell cannot be distinguished. However, a static scattering point results in a systematic deviation of the displacement measurement and is removed (see [5]). While this clutter also occurs in default operation of the GBR, it is much more relevant for the mirror mode. The radar signal takes multiple paths to the corner reflector at the ground and back to the sensor. The first path is the one described above by mirroring the signal at the lower side of the bridge. On a second path the signal reaches the reflector directly. Both paths have different ranges, so they cannot interfere with each other. But when using more than one reflector, it is likely that a direct path from one reflector is in the same range cell as a mirrored path from a different reflector. On the direct path the reflector is static which results in the clutter to be removed as mentioned above.

A direct comparison of the time series requires synchronisation of the different sensor time information. Since this synchronisation cannot be accomplished during the measurement campaign due to hardware restrictions, we address the temporal offset by cross correlating the displacement time series.

Furthermore, the point clouds measured by the profile scanner also have to be processed to compare the GBR data and the profile scanner data directly. The single measurement points are not reproducible and thus have to be spatially clustered [7]. For every cluster, one representative point is calculated by averaging the displacements of the measurement points within the respective cluster. The displacement time series of the representative points result from repeating the processing for every profile scan.

\section{$4 \quad$ Results and discussion}

Figure 5 shows an exemplary comparison of the bridge displacement caused by a truck passing on the bridge. The measurement of the GBR in mirror mode is projected according to Equation (1) to the vertical axis. The second GBR and the profile scanner measure vertical displacement directly and thus can be compared to the data measured by the GBR in mirror mode. The shape of the displacement graph corresponds to a bridge with multiple fields. At first, a positive displacement is measured since the neighbouring bridge field is displaced by the vehicle crossing and lifts the observed field. While the vehicle crosses the observed field the displacement reaches its maximum negative value of $1.5 \mathrm{~mm}$. After the vehicle passage, the bridge oscillates at its eigenfrequency.

Overall, the results of the mirror mode match well with 
Table 1 Sensor specifications.

\begin{tabular}{|c|c|c|c|c|}
\hline Sensor & Model & $\begin{array}{l}\text { Sample } \\
\text { frequency }\end{array}$ & $\begin{array}{l}\text { Precision } \\
\text { range }\end{array}$ & Displacement time series \\
\hline GBR mirror mode & IDS IBIS-FS & $200 \mathrm{~Hz}$ & $<0.1 \mathrm{~mm}$ & Per range cell of $0.75 \mathrm{~m}$ \\
\hline GBR vertical & IDS IBIS-S & $200 \mathrm{~Hz}$ & $<0.1 \mathrm{~mm}$ & Per range cell of $0.75 \mathrm{~m}$ \\
\hline Laser profile scanner & Z+F Profiler 9012 & $50 \mathrm{~Hz}$ & $0.1-0.2 \mathrm{~mm}$ & Per point cluster of $\approx 0.5 \mathrm{~m}$ \\
\hline
\end{tabular}

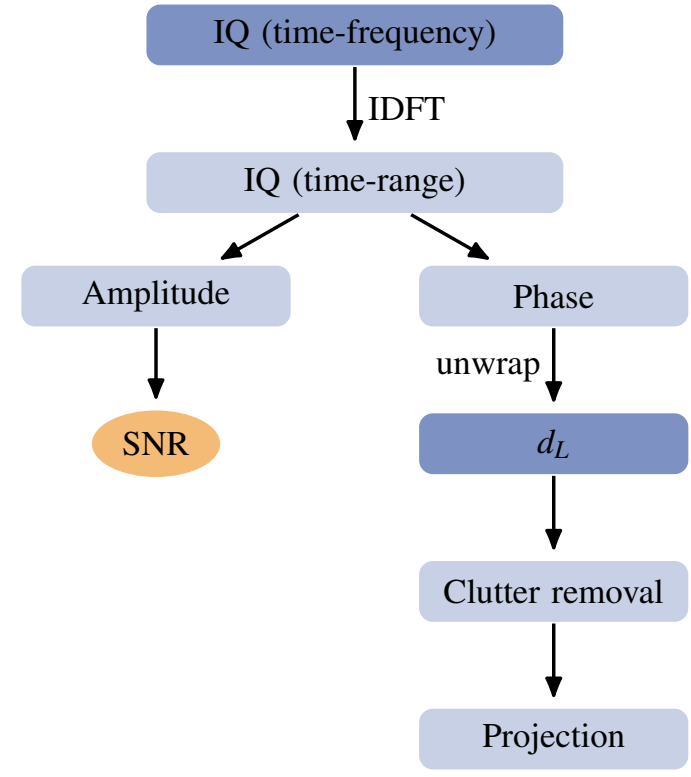

Figure 4 Processing workflow of the GBR data

the other sensors. However, a small deviation in maximum negative displacement can be detected between the two GBR. This can be further analysed by subtracting the displacement in mirror mode from the displacement of the other sensors as shown in Figure 6. The deviation reaches a maximum value of about $-0.1 \mathrm{~mm}$. It is approximately proportional to the absolute displacement as also shown in Figure 7. The difference between the profile scanner and the GBR in mirror mode shows no systematic deviation. It mostly consists of measurement noise from the profile scanner, which is higher than the maximum value of the deviation. Nevertheless this can be an indication that the deviation is caused by the vertical GBR.

The most probable cause for this effect is a difference in the measurement locations. In the default operation the GBR faces the lower side of the bridge at an angle, so that it can measure several points at a time with only range resolution. When facing the lower side of the bridge vertically, the footprint of one resolution cell is quite large. It is therefore possible that slightly different points at the bridge underside are observed. The overall characteristics of the displacement time series would be similar, but a systematic deviation in the absolute values could occur.

Besides comparing the displacement time series, it is important to analyse the possible impact on measurement precision when using the GBR in mirror mode. As the signal is reflected more than once, we expect a lower SNR. In the measurement setup discussed above, the signal of the ver- tical GBR has an estimated SNR of about $60 \mathrm{~dB}$. The SNR of the GBR in mirror mode is estimated at $46 \mathrm{~dB}$. Both are above $35 \mathrm{~dB}$, which is required to achieve the highest possible measurement precision of $0.02 \mathrm{~mm}$ according to [6]. Although the decrease in SNR is acceptable in this measurement campaign, a loss of precision cannot be ruled out for the general purpose.

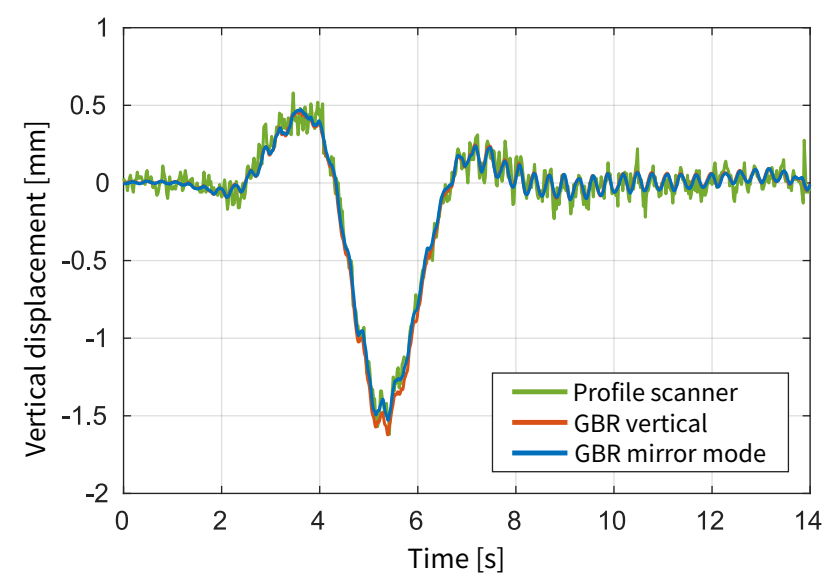

Figure 5 Comparison of the vertical displacement of the bridge for a truck passage measured with all sensors.

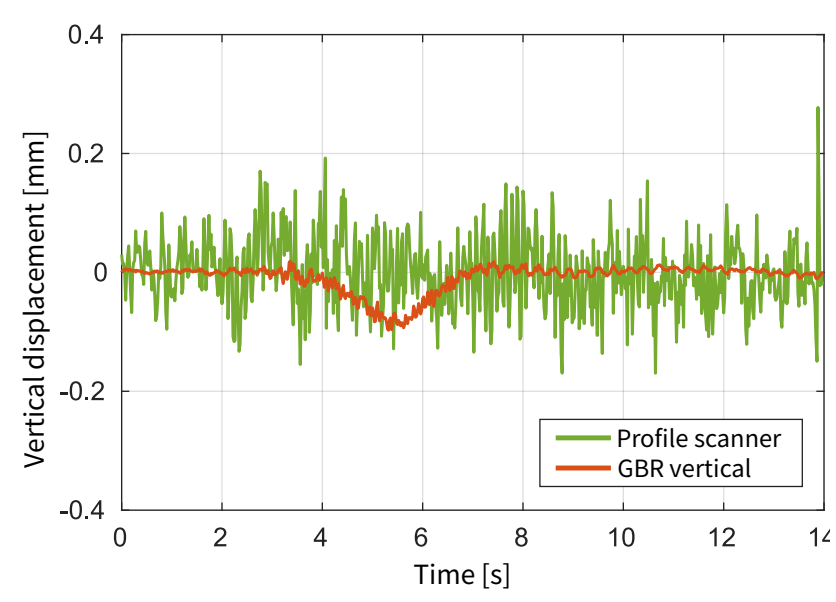

Figure 6 Displacement differences: GBR vertical/Profile scanner subtracted by GBR in mirror mode.

\section{Conclusion and outlook}

This paper addresses the task of monitoring bridge infrastructure with GBR. In contrast to most applied measurement principles, the suggested mirror mode does 


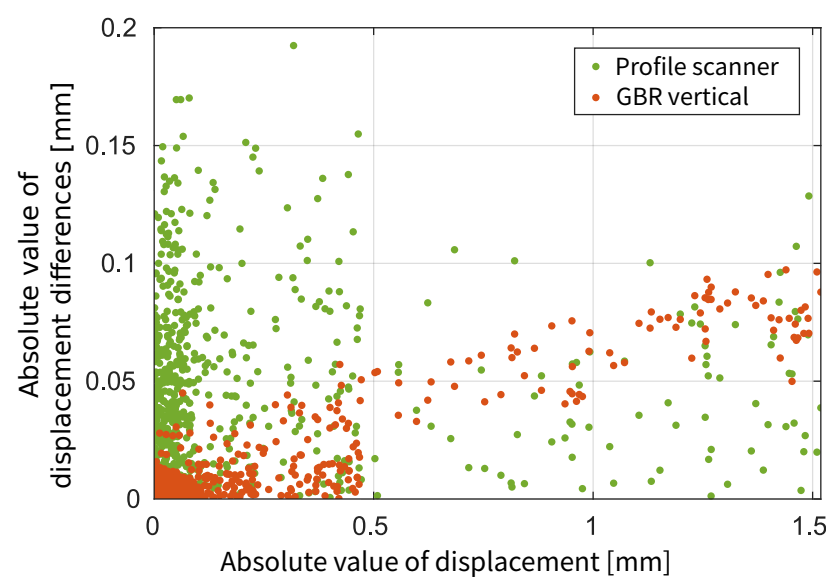

Figure 7 Absolute value of differences as a function of absolute value of displacements.

not rely on scattering points or reflectors at the bridge. Instead this approach uses the flat underside of the bridge as a mirror. One advantage of the mirror mode is a non-invasive measurement by eliminating the need for fixed reflector installation. This reduces setup time and the normal operation of the bridge remains unaffected. It also retains the possibility to have more than one measurement point at the bridge underside unlike a GBR that is placed directly vertically under the bridge. Furthermore, the measurement is unaffected by horizontal displacements of the bridge as only the vertical component is measured. Since the signal is reflected more than once, the SNR of the measurement is generally lower than in default operation of the GBR. Although the decrease in SNR is acceptable in our measurement campaign, this might lead to higher noise for other applications.

We perform the proposed setup successfully in an application at a prestressed concrete bridge. An evaluation is provided by two additional sensors, a second GBR operated in the default configuration and a laser profile scanner. Besides a small systematic deviation between the two GBR, the results of all measurements match well. Along with additional analysis tools, these measurements can now be used to model the bridges condition and observe its behaviour over time.

In future work, we plan to further validate the accuracy of the mirror mode. To determine the cause of the observed deviation, additional comparative measurements with other sensors such as accelerometers will be conducted. Additionally, we will measure other bridge infrastructure with the GBR in mirror mode to be able to generalise the measurement approach.

\section{Acknowledgement}

This work is part of the project ZEBBRA, which is funded by the German Ministry for Education and Research (BMBF). We thank our colleagues of the working group Geodetic Measuring Systems and Sensor Technol- ogy at TU Darmstadt for supporting us with the measurements of the laser profile scanner and we thank the Institute for Automation and Applied Informatics for support with the measurements. We also thank Stefan Hinz for his support.

\section{$7 \quad$ Literature}

[1] Gentile, C. and Bernardini, G.: An interferometric radar for non-contact measurement of deflections on civil engineering structures: laboratory and fullscale tests. Structure and Infrastructure Engineering, 6(5):521-534, 2010.

[2] Pieraccini, M.: Monitoring of Civil Infrastructures by Interferometric Radar: A Review. The Scientific World Journal, 2013.

[3] Pieraccini, M., Parrini, F., Fratini, M., Atzeni, C., Spinelli, P. and Micheloni, M.: Static and dynamic testing of bridges through microwave interferometry. NDT \& E International, 40(3):208-214, 2007.

[4] Dei, D., Mecatti, D. and Pieraccini, M: Static Testing of a Bridge Using an Interferometric Radar: The Case Study of "Ponte degli Alpini," Belluno, Italy. The Scientific World Journal, 2013.

[5] Rödelsperger, S., Läufer, G., Gerstenecker, C. and Becker, M: Monitoring of displacements with groundbased microwave interferometry: IBIS-S and IBIS-L. Journal of Applied Geodesy, 4(1), 2010.

[6] IDS Ingegneria Dei Sistemi S.p.A. Static and dynamic testing of bridges: use of IBIS-FS for measuring deformation and identifying modal analysis parameters. Technical report, 2016.

[7] Schill, F. and Eichhorn, A.: Deformation Monitoring of Railway Bridges with a Profile Laser Scanner. Zeitschrift für Vermessungswesen, 2019. 